\title{
RESEARCH
}

Open Access

\section{Hematopoietic stem cell transplantation for systemic sclerosis: Brazilian experience}

\author{
Álvaro Henrique-Neto1, Marianna Yumi Kawashima Vasconcelos ${ }^{1,2}$, Juliana Bernardes Elias Dias ${ }^{3}$, \\ Daniela Aparecida de Moraes ${ }^{3}$, Maynara Santana Gonçalves ${ }^{2,4}$, Djúlio César Zanin-Silva ${ }^{2,5}$, \\ Talita Graminha Zucoloto ${ }^{1,2,3}$, Marília de Fátima Cirioli de Oliveira ${ }^{3,6}$, Giuliana Martinelli Dotoli, ${ }^{1,2}$, \\ Luiz Fernando Weffort $^{3}$, Vanessa Cristina Leopoldo ${ }^{3,6}$ and Maria Carolina Oliveira ${ }^{2,7^{*}}$ (D)
}

\begin{abstract}
Background: In the past 20 years, hematopoietic stem cell transplantation (HSCT) has been investigated as treatment for systemic sclerosis (SSc). The goal of HSCT is to eradicate the autoreactive immune system, which is replaced by a new immune repertoire with long-lasting regulation and tolerance to autoantigens. Here, we describe the clinical outcomes of severe and refractory SSC patients that underwent HSCT at a single Brazilian center.
\end{abstract}

Patients and methods: This is a longitudinal and retrospective study, including 70 adult SSc patients, with an established diagnosis of SSc, and who underwent autologous HSCT from 2009 to 2016. The procedure included harvesting and cryopreservation of autologous hematopoietic progenitor cells, followed by administration of an immunoablative regimen and subsequent infusion of the previously collected cells. Patients were evaluated immediately before transplantation, at 6 months and then yearly until at least 5-years of post-transplantation followup. At each evaluation time point, patients underwent clinical examination, including modified Rodnan's skin score (mRSS) assessment, echocardiography, high-resolution computed tomography of the lungs and pulmonary function.

Results: Median (range) age was 35.9 (19-59), with 57 (81.4\%) female and median (range) non-Raynaud's disease duration of $2(1-7)$ years. Before transplantation, $96 \%$ of the patients had diffuse skin involvement, $84.2 \%$, interstitial lung disease and 67\%, positive anti-topoisomerase I antibodies. Skin involvement significantly improved, with a decline in mRSS at all post-transplantation time points until at least 5-years of follow-up. When patients with preHSCT interstitial lung disease were analyzed, there was an improvement in pulmonary function (forced vital capacity and diffusing capacity of lung for carbon monoxide) over the 5-year follow-up. Overall survival was $81 \%$ and progression-free survival was $70.5 \%$ at 8-years after HSCT. Three patients died due to transplant-related toxicity, 9 patients died over follow-up due to disease reactivation and one patient died due to thrombotic thrombocytopenic purpura.

(Continued on next page)

\footnotetext{
* Correspondence: mcarolor@usp.br

${ }^{2}$ Center for Cell-Based Therapy, Ribeirão Preto Medical School, University of São Paulo, Ribeirão Preto, Brazil

${ }^{7}$ Department of Internal Medicine, Ribeirão Preto Medical School, University of São Paulo, Avenida dos Bandeirantes 3900, Ribeirão Preto, SP 14048-900,

Brazil

Full list of author information is available at the end of the article
}

(c) The Author(s). 2021 Open Access This article is licensed under a Creative Commons Attribution 4.0 International License, which permits use, sharing, adaptation, distribution and reproduction in any medium or format, as long as you give appropriate credit to the original author(s) and the source, provide a link to the Creative Commons licence, and indicate if changes were made. The images or other third party material in this article are included in the article's Creative Commons licence, unless indicated otherwise in a credit line to the material. If material is not included in the article's Creative Commons licence and your intended use is not permitted by statutory regulation or exceeds the permitted use, you will need to obtain permission directly from the copyright holder. To view a copy of this licence, visit http://creativecommons.org/licenses/by/4.0/. 
(Continued from previous page)

Conclusions: Autologous hematopoietic progenitor cell transplantation improves skin and interstitial lung involvement. These results are in line with the international experience and support HSCT as a viable therapeutic alternative for patients with severe and progressive systemic sclerosis.

Keywords: Systemic sclerosis, Hematopoietic stem cell transplantation, Stem cells, Progenitor cells, Transplantation

\section{Background}

Autologous hematopoietic stem cell transplantation (HSCT) has been investigated as treatment for systemic sclerosis (SSc) for more than two decades, with progress both in choosing the most suitable candidates and in reducing the morbidity and mortality associated with the procedure [1-3]. Patients with severe and rapidly progressive SSc have been enrolled for HCST, and according to data from international transplant registries, the number of transplanted patients per year has progressively increased, reflecting the good outcomes of this therapeutic approach [4].

The initial phase I/II studies were important to establish recommendations and to guide currently adopted transplant procedures [5-12]. Over time, details about patient selection, drugs included in the procedure, patient management and follow-up were discussed and improved around multiple transplantation centers. These studies also evidenced the importance of a proper heart assessment as a strategy to decrease transplant-related mortality. Cardiac dysfunctions that occur during transplantation accounted for many of the transplant-related deaths described in the initial studies [13]. Currently, recommendations for pre-transplantation cardiac assessment are available in the literature [14]. Multivariate analyses have also shown that the experience of the center in transplanting autoimmune diseases has also a significant impact on patient outcomes. Centers with more than 26transplanted autoimmune disease patients and those with more than 6 years of experience have better disease outcomes over time than less experienced centers [4].

The goal of transplantation is to non-selectively eliminate autoreactive $\mathrm{T}$ and B-lymphocytes through high-dose immunosuppression and to reconstitute a new immunological system by infusion of autologous hematopoietic progenitor cells, previously collected and cryopreserved [15]. The strategy promotes an immunological resetting, in which the existing immune system is eradicated, and replaced by a new immune repertoire with adequate longlasting regulation and tolerance to autoantigens $[16,17]$. Studies have described thymic reactivation, increased frequencies of cells with regulatory function, improvement of T-cell receptor diversity and normalization of molecular signatures as post-transplantation mechanisms associated with clinical outcomes $[18,19]$.

Autologous HSCT has a striking impact on skin involvement, as mRSS significantly declines, starting in the first months after the procedure $[2,5,20]$. Pulmonary function also benefits from transplantation, in most cases stabilizing or, less frequently, improving after HSCT $[2,5,20]$. To date, three prospective and randomized studies have shown that autologous HSCT is superior to conventional therapy with monthly cyclophosphamide infusions in patients with severe and progressive SSc [21-23]. These studies report higher overall survival and progression-free survival rates, as well as better disease control and quality of life in transplanted versus non-transplanted patients. A recent meta-analysis shows that autologous HSCT is superior to conventional therapies in SSc patients, especially in the analyses of risk of death from any cause, skin and lung involvement, and quality of life [24]. Recently, the European League Against Rheumatism (EULAR), the American Society for Transplantation and Cell Therapy (ASCTC) and the Brazilian Society of Bone Marrow Transplantation (SBTMO) have recommended autologous HSCT as treatment for patients with progressive SSc at risk of organ failure $[3,25,26]$.

The present study aims to evaluate the outcomes of severe and progressive SSc patients treated with autologous HSCT at a single Brazilian center, and to compare them with the international experience.

\section{Patients and methods}

\section{Study design and patient enrolment}

This retrospective study included 70 SSc patients older than 18 years of age, diagnosed according to the ACR criteria of 1980 [27] and ACR/EULAR of 2013 [28], who underwent autologous HSCT from November 2009 to May 2016. The institutional ethics committee approved the study (CAAE: 71204717.6.0000.5440) and consent forms were waved due to the retrospective nature of data collection. At enrolment for transplantation, every patient had signed informed consent forms agreeing to participate in HSCT as an experimental protocol, with collection of clinical data and biological samples for the purpose of research.

Patients were eligible for transplantation if up to 60 years of age, and with evidence of skin and/or interstitial lung disease worsening in the past 6 months, despite immunosuppressive therapy. Specifically, transplant indications included diffuse skin involvement with increases in the mRSS [29] by more than 25\% (if initial mRSS greater than 14) and/or interstitial lung disease with worsening 
of pulmonary function detected by reduction in forced vital capacity (FVC) and/or diffusing capacity of lung for carbon monoxide (DLCO) predicted values greater than $10 \%$. Exclusion criteria for the procedure were: left ventricular ejection fraction below $40 \%$, constrictive pericarditis, moderate or severe diastolic dysfunction (evidenced by echocardiography, cardiac MRI or right side catheterism) or ventricular arrhythmias, FVC below $45 \%$, DLCO lower than $40 \%$, systolic pulmonary artery pressure above $40 \mathrm{mmHg}$ by echocardiography or mean pulmonary artery pressure higher than $27 \mathrm{mmHg}$ by right side catheterism, serum creatinine levels higher than $2 \mathrm{mg} / \mathrm{dl}$, aspartate aminotransferase/ alanine transaminase (AST/ALT) serum levels above 3 times the normal limit, total bilirubin serum levels greater than $2 \mathrm{mg} /$ $\mathrm{dl}$ (excluding Gilbert's disease), positive serology for HIV or HTLV 1 and 2, previous malignancy, positive pregnancy test, psychiatric disorder and cognitive impairment that compromises treatment adherence or understanding of the consent form. Overlapping autoimmune diseases did not contraindicate enrolment for the procedure.

\section{Experimental procedure and data collection}

Immediately before transplantation, all patients underwent a thorough clinical and laboratorial assessment, aiming to evaluate the disease status, as well as possible exclusion conditions. Rodnan's skin score was measured by an experienced rheumatologist. Patients collected blood and urine samples for complete blood counts, biochemical profile, infectious serologies, pregnancy tests (female patients), urine analysis, creatinine clearance, proteinuria, and autoantibody assays. Patients underwent pulmonary function tests, high resolution computed tomography of the lungs, esophageal manometry, $24 \mathrm{~h}$ electrocardiography (Holter) and echocardiography. Patients transplanted after the year 2014 also completed cardiac magnetic resonance and right-side cardiac catheterism with fluid challenge (infusion of $500 \mathrm{ml}$ of saline over $10-15 \mathrm{~min}$ ) as part of the pre-transplantation screening.

Autologous HSCT comprised a first phase, consisting of mobilization of hematopoietic progenitor cells from the bone marrow into the peripheral blood through infusion of cyclophosphamide at a dose of $2 \mathrm{~g} / \mathrm{m}^{2}$ and granulocyte colony stimulating factor (G-CSF), followed by apheresis and cryopreservation of unmanipulated hematopoietic progenitor cells (aiming for more than $2 \times 10^{6} \mathrm{CD} 34+$ cells $/ \mathrm{kg}$ of patient body weight). After a minimum interval of 2 weeks, patients started the second phase, comprising intense immunoablation, followed by infusion of the previously collected cells (Fig. 1). The standard immunoablative regimen consisted of $200 \mathrm{mg} /$ $\mathrm{kg}$ cyclophosphamide plus $4.5 \mathrm{mg} / \mathrm{kg}$ anti-thymocyte globulin (ATG). When patients were overweight, cyclophosphamide doses were adjusted for ideal body weight (calculated considering ideal body mass index of $25 \mathrm{~kg} /$ $\mathrm{m}^{2}$ ). For patients with evidence of heart involvement, but not fulfilling exclusion criteria for transplantation, the regimen consisted of fludarabine $120 \mathrm{mg} / \mathrm{m}^{2}$ and melphalan $120 \mathrm{mg} / \mathrm{m}^{2}$ of body surface plus $4.5 \mathrm{mg} / \mathrm{kg}$ ATG. Autologous progenitor cells infusions were followed by approximately 7 days of aplasia (destruction of the previous immuno-hematological system), and subsequent recovery of red blood cells, leukocytes and platelets. Neutrophil and platelet engraftment was considered when the patients reached at least 500 neutrophils $/ \mu \mathrm{L}$ and at least 20.000 platelets $/ \mu \mathrm{L}$ for three consecutive days, respectively, not supported by transfusion.

Patients were evaluated immediately before transplant (pre-HSCT), at 6 months and then yearly, until at least 60 months of follow-up after transplantation, after which patients returned to their original rheumatologist. At each evaluation, patients underwent clinical examination, including mRSS, complete blood counts, biochemical tests, high-resolution computed tomography of the chest and pulmonary function with DLCO. Clinical data were obtained from medical records. Disease progression/reactivation was considered when there was worsening of mRSS values above $25 \%$ of baseline (pretransplant) levels, decline in FVC or DLCO predicted percentages above $10 \%$, scleroderma renal crisis or initiation of immunosuppressive therapy. A HSCT failure would be established if the patient fulfilled the progression criteria at the first ( 6 months) evaluation after transplant. None of the patients had a HSCT failure. Beyond the fifth year of post-HSCT follow-up, monitoring for survival and late disease reactivation was done by telephone calls, whenever possible.

\section{Statistical analysis}

An exploratory data analysis was performed using measures of central position, dispersion and box-plot graphs for quantitative variables. Qualitative variables are presented considering absolute and relative frequencies. Linear regression models of mixed effects were performed to verify the effect of time in relation to the variables where the repeated measures of each patient were considered as a random effect and time as a fixed effect. Comparisons between evaluation periods were performed using orthogonal contrasts. The fit of the model was verified through the analysis of residues. Statistical analyses used SAS software (SAS Institute Inc., NC, USA) version 9.4. Graphs were created with GraphPad Prism (GraphPad Software, Inc., CA, EUA) version 9. Significance was set at 0.05 . 


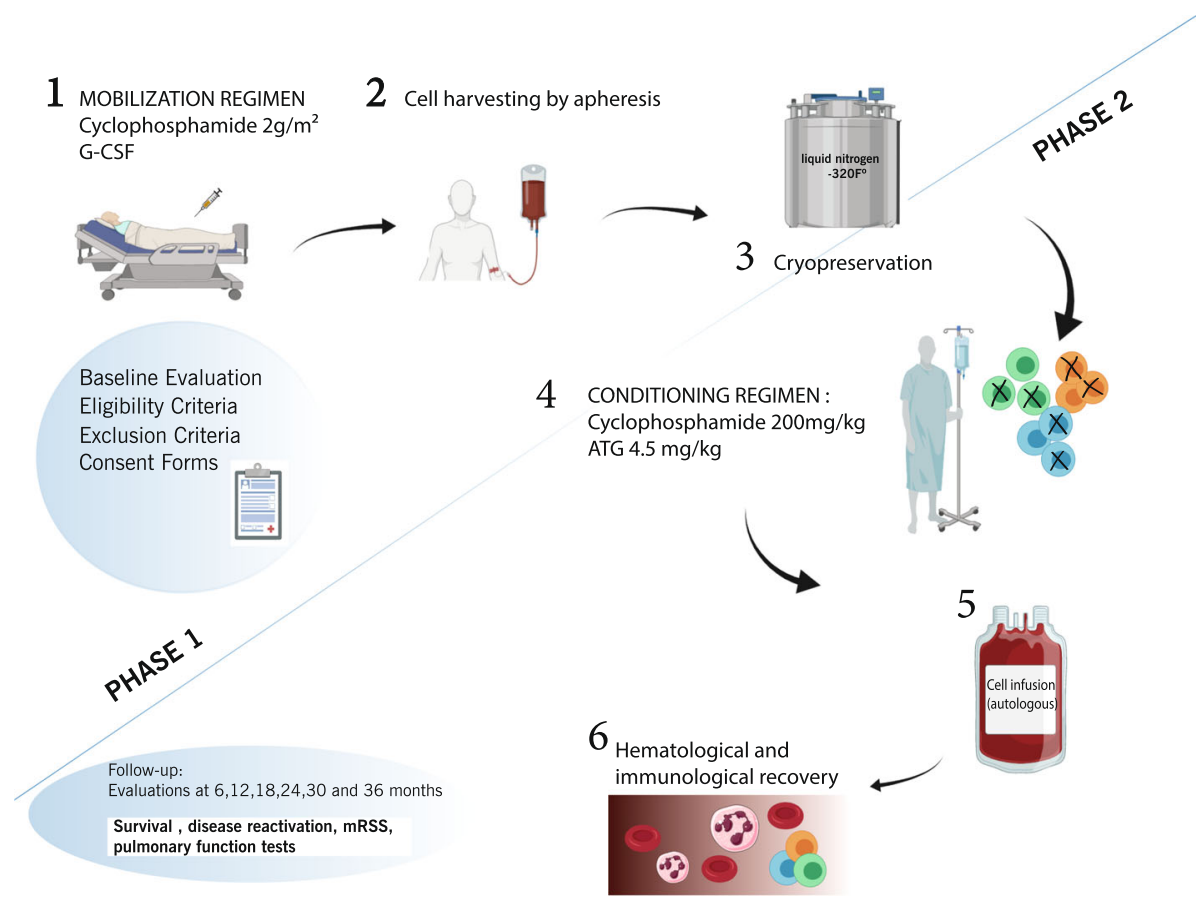

Fig. 1 Study design. Hematopoietic stem cell transplantation comprises two phases. In phase I, patients that fulfill the inclusion criteria for transplantation are thoroughly evaluated for organ involvement, eligibility and exclusion criteria (baseline evaluation). Then, progenitor hematopoietic cells (CD34+) are mobilized from the bone marrow to the peripheral blood by a regimen of cyclophosphamide plus granulocytecolony stimulating factor (G-CSF). Circulating CD34+ cells are harvested from the peripheral blood through apheresis and cryopreserved, without further manipulation. In phase 2, which begins at least 2 weeks after phase 1, patients are treated with a conditioning regimen consisting of combined high-dose chemotherapy and immunotherapy agents, usually cyclophosphamide $200 \mathrm{mg} / \mathrm{kg}$ plus anti-thymocyte globulin (ATG). Subsequently, the previously harvested autologous progenitor cells are thawed at bedside and administered to the patient, intravenously. A period of bone marrow aplasia follows, lasting approximately 7 days, ended by hematological and immunological recovery. Patients are followedup periodically for hematological and immunological reconstitution and clinical outcomes, until 5-years of follow-up

\section{Results}

Seventy transplanted adult SSc patients were initially included in the study, and 66 patients had available data for analysis on long-term follow-up (Fig. 2). Table 1 shows baseline (pre-HSCT) characteristics of all adult transplanted patients. Most subjects presented diffuse SSc, had interstitial lung disease and gastrointestinal involvement. Approximately two thirds of the patients had positive anti-topoisomerase I (anti-SCl70) antibodies and all had been treated with at least one line of immunosuppressive therapy. Fifty-one (73\%) patients had progressing interstitial lung disease as indication for HSCT, out of which 46 (90\%) had been previously treated with intravenous cyclophosphamide, 11 (21\%) with mycophenolate mofetil and $4(8 \%)$ with rituximab.

\section{Immediate transplant outcomes}

Sixty-nine patients underwent mobilization with cyclophosphamide and G-CSF, as determined by institutional protocol. One patient was mobilized with G-CSF only, due to a previous episode of acute cardiac insufficiency after a standard cyclophosphamide pulse. Mean (standard deviation, SD) CD34+ cell count was $5.63(3.21) \times$
$10^{6}$ cells per kilogram of body weight and there were no progenitor harvesting failures. Sixty-five (92.8\%) patients received immunoablative conditioning regimen with cyclophosphamide and ATG. The five remaining patients were treated with the fludarabine plus melphalan and ATG regimen, due to cardiac involvement by SSc evidenced by the pre-transplantation screening. All patients presented neutrophil and platelet engraftment at median (range) time of 9 (8-11) and 10 (9-14) days after HSCT, respectively. For the subset of patients transplanted with fludarabine and melphalan, median (range) time for neutrophil engraftment was of 11 (1013) days, therefore slightly delayed in comparison to the whole group of patients. Platelet engraftment was not different.

Gastrointestinal manifestations, mostly nausea and vomiting, as well as alopecia, are expected side effects of cyclophosphamide and occurred in all patients (Table 2). Acute myocardial toxicity, verified by echocardiography as a decrease in left ventricle ejection fraction of more than $10 \%$ and increase in serum troponin levels, was detected in four of the patients treated with high-dose cyclophosphamide. Most patients presented fever while 


\section{TRANSPLANTED PATIENTS}

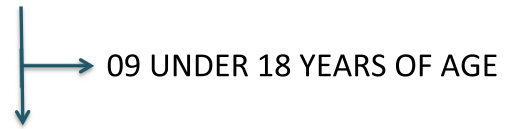

\section{ADULT PATIENTS}

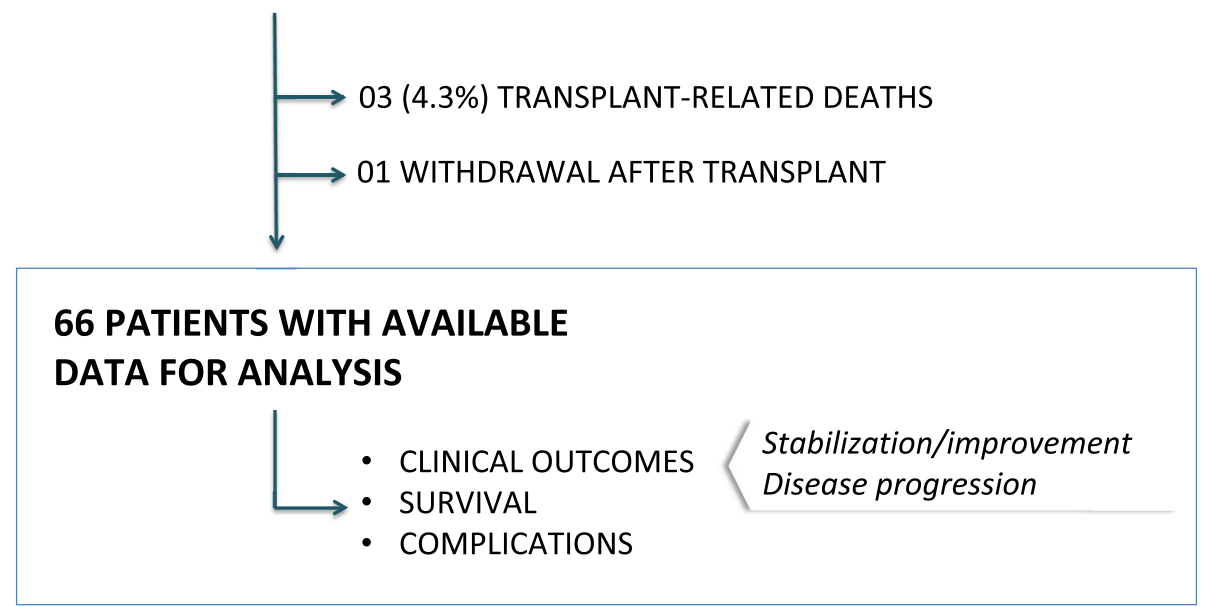

Fig. 2 Number of patients included in the retrospective study. From the initial 79 patients transplanted from 2009 to 2016, nine were under 18 years of age and were therefore excluded. Three patients died due to transplant-related complications (sepsis, complications of acute acalculous cholecystitis and acute cyclophosphamide-induced cardiotoxicity) and therefore did not have follow-up data. One patient voluntarily left the study after transplantation and was lost to follow-up (alive in December 2019, as confirmed by phone call)

in the neutropenic phase of HSCT, but infections were clinically identified in only $8(11 \%)$ of the patients. After engraftment, cytomegalovirus (CMV) reactivations were detected by polymerase chain reaction in $10(14 \%)$ of the patients in the immediate post-transplantation period (up to 60 days after HSCT), and were more frequent in patients treated with fludarabine and melphalan (4 out of 5 patients) than in the cyclophosphamide-treated patients ( 6 out of 62). None of the patients developed clinical manifestations of CMV disease. Two patients who had systemic lupus erythematosus (SLE) overlapping with the SSc presented improvement of both autoimmune diseases after HSCT, however some manifestations of SLE (hemolytic anemia and thrombocytopenia in one patient, and pericarditis in the other) reactivated approximately 24 months after transplant.

\section{Survival}

At 3, 5 and 8-year follow-up, the overall survival rates (95\% confidence interval, CI) were 91.8\% (81.2-97.1), $85.9 \% \quad(73.8-93.4)$ and $81 \%(64.3-89.1)$ and the progression-free survival rates $(\mathrm{CI})$ were $71.8 \%(61.1-$ $83.4), 71.8 \%(60.1-82.9)$ and $70.5 \%$ (59.2-81.4), respectively (Fig. 3). There were three transplant-related deaths within 100 days after HSCT. One patient with extensive gastrointestinal involvement at baseline died 23 days after autologous HSCT due to sepsis by Acinetobacter baumannii. A second patient with advanced disease, but no evidence of cardiac involvement at enrolment died 60 days post-transplantation due to complications after an episode of acute cyclophosphamide-induced cardiac toxicity. A third patient presented an acute episode of acalculous cholecystitis and required emergency laparotomy during transplantation, while still in the neutropenic phase. In the post-operatory period, she presented a mixed septic and cardiogenic shock, which led to death 1 week after HSCT. Over long-term follow-up, nine additional patients died due to disease reactivation and organ failure, mostly cardiopulmonary, and one patient died due to central nervous system complications of thrombocytopenic thrombotic purpura (described in Table 2).

\section{Disease outcomes}

Before HSCT, median (range) mRSS was 24 (8-51), and significantly improved after the procedure, from 6 months until the 5-year follow-up time point (Fig. 4a). Forced vital capacity (Fig. 4b) and DLCO (Fig. 4c) percentages stabilized after HSCT in the 66 SSc patients that had follow-up data after HSCT. However, when separately analyzed, the subgroup of 51 patients with progressing interstitial lung disease (presenting more than $10 \%$ decline in FVC or DLCO in the 6 months preceding enrolment for HSCT) presented improvements in both FVC and DLCO predicted percentages after HSCT (Fig. 4d and e). In total, 17 patients had disease 
Table 1 Patient baseline characteristics and transplant details

\begin{tabular}{ll}
\hline Total number of adult patients & 70 \\
Median (range) age (years) & $35.9(19-59)$ \\
Gender (\%) & $57(81.4 \%)$ female \\
Disease subtype & $67(95.7 \%)$ diffuse \\
Median (range) disease duration (years) ${ }^{\mathrm{a}}$ & $2(1-7)$ \\
Organ involvement: & \\
Skin n (\%) & $70 / 70(100 \%)$ \\
Interstitial lung disease, n (\%) & $59 / 70(84.2 \%)$ \\
Pulmonary hypertension, n (\%) & $4 / 70(5.7 \%)$ mild \\
Heart involvement, n (\%) & $32 / 70(45.7 \%)^{\mathrm{b}}$ \\
Gastrointestinal, $\mathrm{n}(\%)$ & $57 / 62(92 \%)$ \\
Kidney n & 0 \\
Positive anti-SCl70 antibodies, n (\%) & $43 / 64(67.2 \%)$ \\
Previous immunosuppressive treatment: & \\
Methotrexate, n (\%) & $26 / 70(37.1 \%)$ \\
Cyclophosphamide, n (\%) & $48 / 70(68.6 \%)$ \\
Mycophenolate mofetil, n (\%) & $13 / 70(18.6 \%)$ \\
Rituximab, n (\%) & $4 / 70(5.7 \%)$ \\
Transplant regimen: & \\
Cyclophosphamide + ATG & $65(92.8 \%)$ \\
Fludarabine + Melphalan + ATG & $5(7.2 \%)$ \\
\hline
\end{tabular}

Baseline (pre-transplant) clinical characteristics of patients enrolled in the study

$I V$ intravenous infusion, ATG anti-thymocyte globulin

${ }^{a}$ Disease duration from first non-Raynaud's phenomenon clinical manifestation until transplant

${ }^{b}$ Patients with severe heart involvement were excluded from transplant

reactivation after HSCT, 15 of which required further immunosuppressive treatment (Table 2). Worsening of the interstitial lung disease, verified by high-resolution computed tomography and decline in pulmonary function, was the most frequently detected reactivation, usually treated with rituximab and/or mycophenolate mofetil. Skin and myopathy reactivations were treated with methotrexate, and gastrointestinal worsening was treated with prokinetic agents and nutritional support. Two patients required jejunostomies for feeding.

\section{Discussion}

Hematopoietic stem cell transplantation for autoimmune diseases has been practiced for at least 20 years worldwide. To date, more than 2000 transplants have been reported by tenths of transplant centers [4]. At our center, the experience with HSCT has begun in 2001, with an initial focus on systemic lupus erythematosus and multiple sclerosis [30,31]. Later, we started to consistently enroll patients with severe and progressive SSc. Here, we describe the clinical outcomes of 70 adult SSc patients treated at a single center, from 2009 and 2016, under similar transplant protocols.
Most patients in our study presented progressive interstitial lung involvement as indication for HSCT. When the whole cohort of patients was analyzed, we observed stabilization of pulmonary function, which agrees with most international studies [2, 5, 20-28]. However, when the subgroup of patients with progressive interstitial lung disease as indication for HSCT was analyzed separately, we were able to detect significant improvement of FVC and DLCO after HSCT. In accordance, a systematic review of the literature compiled data from the main clinical trials and found improvement in FVC at 1 and 2 years after HSCT [32].

Recently, we have reported quantitative computed tomography outcomes of our SSc patients, showing that in patients that improve lung function the volume of diseased lung parenchyma decreases after HSCT, when compared to pre-transplant measurements [33]. We believe that functional improvement reflects a control in the inflammatory component of interstitial lung disease, which is an early manifestation of diffuse SSc. Indeed, some authors suggest that patients should be transplanted early in disease course, aiming to achieve higher reversibility of organ involvement $[21,22]$. In our study, the median disease duration of our patients, at enrolment, was 2 years, indicating that at least half were enrolled still at early stages of disease. On the other hand, we included patients up to 7 years of disease duration, but duration of disease did not strongly correlate with skin and lung function outcomes (data not shown), indicating that other factors, yet to be determined, might be participating.

The most clinically detectable outcome of HSCT is the improvement of skin thickness, assessed by mRSS. This is a consistent finding across studies on HSCT for SSc, as skin thickness decreases significantly, especially in the first 6 months after HSCT [1, 2, 5, 20, 24, 32]. Rodnan's score has good correlation with dermal thickness, evaluated both through skin biopsies and ultrasound [34, 35]. In fact, histopathological analyses show a decline in dermal thickness at 1 year post-transplantation, which is not different from that of healthy controls at 5 years after HSCT [5]. An interesting aspect is that most patients in our cohort had been previously treated with intravenous cyclophosphamide, and yet had a good response to HSCT using high-dose cyclophosphamide. We were not able to observe differences in outcomes according to previous therapy, and even if cyclophosphamide is not usually indicated for skin involvement, we did observe considerable improvement of mRSS after HSCT.

Skin involvement in SSc has a negative impact on survival and on quality of life [36]. A previous study from our group, which included a subset of patients also enrolled in this present study, has shown that autologous HSCT improves functional outcomes of SSc patients, 
Table 2 Complications and disease-related events after transplantation

\begin{tabular}{|c|c|}
\hline Transplant-related complications $^{a}$ & N (\%) \\
\hline Nausea/vomiting & $70(100 \%)$ \\
\hline Alopecia & $70(100 \%)$ \\
\hline Neutropenic fever & $65(93 \%)$ \\
\hline Bacterial pneumonia & $5(7 \%)$ \\
\hline Cyclophosphamide-induced cardiac dysfunction & $4(6 \%)$ \\
\hline Sepsis & $2(3 \%)$ \\
\hline Melphalan-induced mucositis & $2(3 \%)$ \\
\hline Central catheter-related pneumothorax & $1(1 \%)$ \\
\hline Acalculous cholecystitis & $1(1 \%)$ \\
\hline Polyomavirus-induced hemorrhagic cystitis (mild) & $1(1 \%)$ \\
\hline CMV reactivation (no CMV disease) & $10(14 \%)$ \\
\hline Transplant-related deaths (total): & $3(4 \%)$ \\
\hline \multicolumn{2}{|l|}{ Sepsis, Acinetobacter baumannii } \\
\hline \multicolumn{2}{|l|}{$\begin{array}{l}\text { Complications of acalculous cholecystitis } \\
\text { Acute myocardial toxicity }\end{array}$} \\
\hline \multicolumn{2}{|l|}{ Disease reactivations } \\
\hline Skin worsening & $3(4 \%)$ \\
\hline Pulmonary function worsening & $6(9 \%)$ \\
\hline Inflammatory myopathy & $2(3 \%)$ \\
\hline Gastrointestinal progression/gastroparesis & $4(6 \%)$ \\
\hline Other (SLE reactivation, cytopenia) requiring immunosuppression & $2(3 \%)$ \\
\hline Disease-associated deaths (total): & $9(13 \%)$ \\
\hline \multicolumn{2}{|l|}{ Acute lung exacerbation (3 patients) } \\
\hline \multicolumn{2}{|l|}{ Gastrointestinal progression (2 patients) } \\
\hline \multicolumn{2}{|l|}{ Cardiac insufficiency (2 patients) } \\
\hline \multicolumn{2}{|l|}{ Scleroderma renal crisis (1 patient) } \\
\hline \multicolumn{2}{|l|}{ Pulmonary embolism (1 patient) } \\
\hline \multicolumn{2}{|l|}{ Non-disease related events } \\
\hline Thrombotic thrombocytopenic purpura (death) & $1(1 \%)$ \\
\hline Pregnancies in female transplanted patients & $3(5.5 \%)$ \\
\hline
\end{tabular}

Number of complications associated or not associated with the transplant procedure, including disease reactivation and long-term survival outcomes CMV cytomegalovirus, SLE systemic lupus erythematosus (disease overlap)

${ }^{a}$ Complications associated to the procedure within 60 days after HSCT

including functional capacity measured by the 6-min walking distance, inspiratory and expiratory lung pressures, joint range-of-motion, hand function and mouth opening, as well as physical and mental aspects of quality of life [37]. We consider important to evaluate such outcomes, as they reflect the positive impact of HSCT on the daily life of patients. Although most of these functional assessments are closely related with skin involvement, functional capacity did not strongly correlate with pulmonary, skin or musculoskeletal outcomes, suggesting that the improvement is a combination of multiple effects of HSCT.

Patient outcomes after HSCT for autoimmune diseases have considerably improved over the years. Nevertheless, a number of adverse events associated with the procedure still persist. Systemic sclerosis, among the autoimmune diseases, has the highest transplant-related morbidity and mortality rates $[4,38]$, mostly due to baseline organ involvement by the disease. Our study reports three transplant-related deaths. In at least two of these events, patients were very ill and severely affected by the disease. In our retrospective perspective, more stringent patient selection might have avoided the fatal outcomes. Systemic sclerosis has a very narrow window of opportunity for HSCT and patients at end-stage disease should not be enrolled. This is part of the "learning curve" that follows medical and scientific progress [4, 38]. Fortunately, advances in patient selection and 

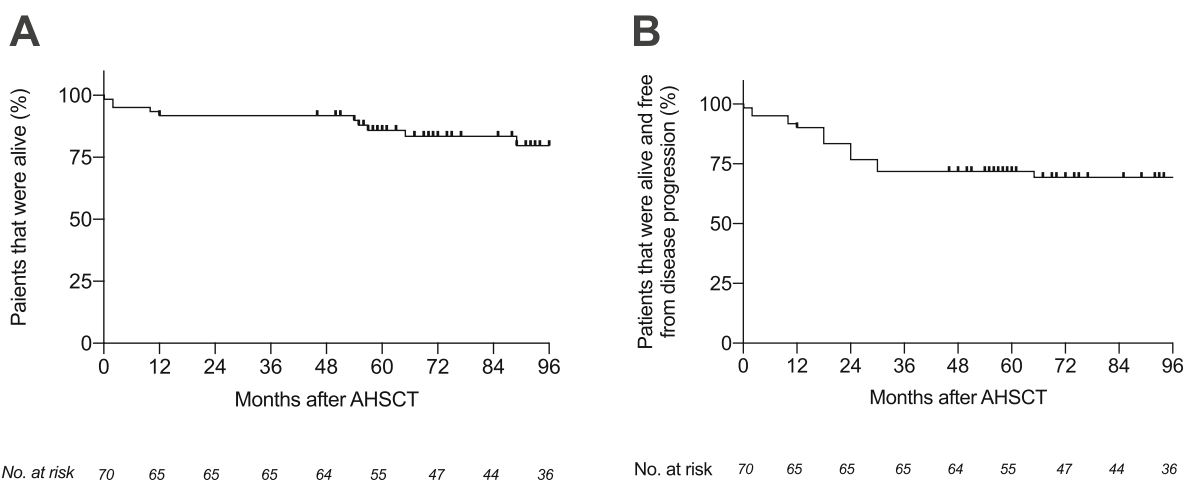

Fig. 3 Survival outcomes over 8-year follow-up after transplantation. a Overall survival; percentage of patients that were alive at each yearly follow-up time point. b Progression-free survival; percentage of patients that were alive and free from disease progression at each follow-up time point. Disease progression criteria are described in the Patients and Methods section. The numbers of patients at risk, at each time point, are described below each graph

management have enabled better survival rates and outcomes over the years [38].

Cardiac involvement plays a key role in patient safety, as SSc affects the heart in multiple and silent manners [20, 39]. Standard pre-transplantation evaluations with electro and echocardiography are not sufficiently accurate to identify SSc patients at risk during HSCT [20]. The combination of high dose cyclophosphamide, fluid overload and cytokine release inflicts excessive strain on the already dysfunctional cardiopulmonary system and may impose risks. Recently, the EBMT and partners have published recommendations for pre-transplantation evaluations of SSc patients [14]. In fact, at our center, cardiac failure events have declined, albeit not completely ceased, after we started to follow such guidelines, including cardiac MRI and right side catheterism with fluid overload in our pre-transplantation evaluation. In addition, in cases where the patient presented some
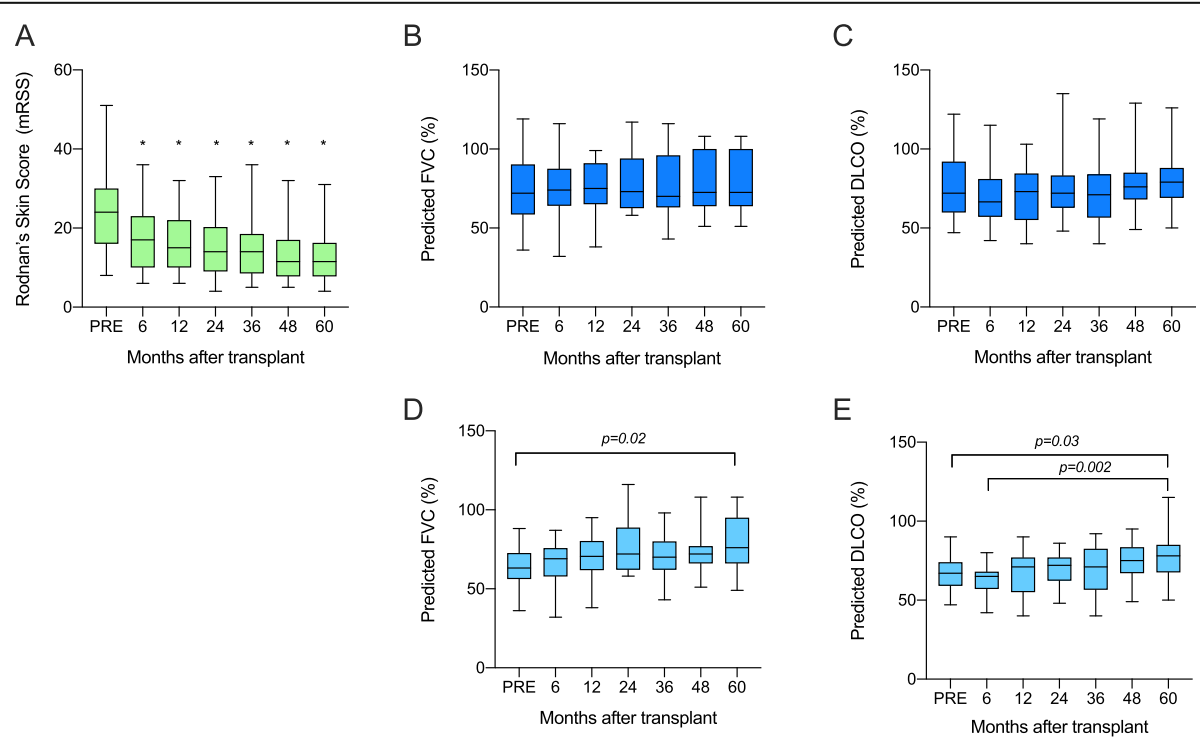

Fig. 4 Clinical Outcomes over 5-year follow-up after transplantation. a Modified Rodnan's Skin Score before and periodically after autologous hematopoietic stem cell transplantation (HSCT). ${ }^{*}$ indicates significant diferences between baseline (PRE) and post-HSCT time points: PRE vs 6 months, $p=0.007$; PRE vs 12 months, $p=0.0006$; PRE vs 24, 36, 48 and 60 months, $p<0.0001$. b Predicted forced vital capacity (FVC) percentages before and periodically after autologous HSCT in all patients included in the study. c Predicted percentages of diffusing capacity of lung carbon monoxide (DLCO) before and periodically after autologous HSCT in all patients included in the study. $\mathbf{d}$ Predicted FVC percentages before and periodically after autologous HSCT in the subset of patients with progressive interstitial lung disease at enrollment for transplantation. e Predicted percentages of diffusing capacity of lung carbon monoxide (DLCO) before and periodically after autologous HSCT in patients with progressive interstitial lung disease at enrollment for transplantation. Box plot limits indicate 25th to 75th percentiles; lines within boxes indicate the median and whiskers mark the 10th and the 90th percentiles 
degree of cardiac damage, but not formally fulfilled the exclusion criteria, we chose an alternative transplant regimen, with fludarabine plus melphalan, instead of high dose cyclophosphamide. Although only a few patients have been included in this new regimen, we have observed a good profile of cardiac safety (data not shown), despite a possible increase in the incidence of mucositis and infectious events such as CMV reactivation. The number of patients transplanted with this alternative regimen is not large enough to enable comparisons regarding long-term efficacy in controlling disease progression. A similar strategy has been recently published, aiming to decrease the total dose of cyclophosphamide in patients with high SSc-associated cardiac risk [40].

The patients enrolled in our study have a severe disease profile, as most have diffuse skin involvement, positive anti-SCl 70 antibodies and progressive interstitial lung disease. We also included patients with overlapping SLE, and although SLE is also a primary indication for HSCT [4], it adds complexity and risk to the procedure. Patients with similar characteristics are also described by most trials on HSCT for SSc [1, 2, 20-23]. In face of the severity of the disease, these patients have benefited from HSCT, despite the toxicity of the procedure. On the other side, transplant-related mortality is closely related to the degree of organ involvement by the disease, especially cardiopulmonary and gastrointestinal [20,38]. Therefore, adequate patient selection is key to ensure enrollment within the adequate window of opportunity. The main challenge is to identify patients with a disease severe enough to require HSCT, but still not too advanced in order to tolerate transplant-related toxicity. In this context, scientific dissemination and educational events are essential to increase rheumatologist awareness that HSCT may be an option for patients with the right profile of disease.

\section{Conclusions}

In this study, we show a Brazilian experience of HSCT as a therapeutic strategy for patients with SSc. The survival and disease outcomes here described are in accordance with those reported by other international transplant centers. Conventional treatment options for SSc have still limited efficacy in promoting disease control, and HSCT stands as an alternative for selected patients with severe and progressive disease.

\section{Abbreviations}

AST/ALT: Aspartate aminotransferase/alanine transaminase; ASTCT: American Society for Transplant and Cell Therapy; ATG: Anti-thymocyte globulin; Cl: Confidence interval; CMV: Cytomegalovirus; DLCO: Diffusing capacity for carbon monoxide; EULAR: European League Against Rheumatism;

FVC: Forced Vital Capacity; G-CSF: Granulocyte-colony stimulating factor; HIV: Human immunodeficiency virus; HSCT: Hematopoietic stem cell transplantation; HTLV: Human T-lymphotropic virus; mRSS: Modified Rodnan's
Skin Score; SBTMO: Brazilian Society of Bone Marrow Transplantation; SD: Standard Deviation; SSc: Systemic sclerosis

\section{Acknowledgements}

The authors thank the multidisciplinary team of the Hospital das Clínicas da Faculdade de Medicina de Ribeirão Preto - Universidade de São Paulo for their work in patient care and also the Ribeirão Preto Regional Blood Center staff for processing and cryopreservation of the progenitor hematopoietic cells. Their contributions have made this manuscript possible.

\section{Authors' contributions}

$\mathrm{AHN}$ - made significant contributions to acquisition, interpretation and analysis of the data, and drafted the manuscript; MYKV, DAM, JBED, TGZ, MFCO and LFW contributed to data acquisition; MSG, DCZS, GMD and VCL contributed to the analysis and interpretation of the data; MCO designed the study, supervised data acquisition, interpretation and analysis and substantially revised the manuscript. All authors approve the submitted version of this manuscript. All authors agree both to be personally accountable for the author's own contributions and to ensure that questions related to the accuracy or integrity of any part of the work, even ones in which the author was not personally involved, are appropriately investigated, resolved, and the resolution documented in the literature.

\section{Funding}

This study has been partially funded by the São Paulo Research Foundation (FAPESP) grant \#2013/08135-2 and by the Coordenação de Aperfeiçoamento de Pessoal de Nível Superior - Brasil (CAPES) - Finance Code 001. The graduate students that co-author this manuscript have received fellowships and from FAPESP (MYKV), CAPES (MSG, TGZ, GMD), and National Council for Scientific and Technological Development - CNPq (DCZS).

\section{Availability of data and materials}

The datasets used and/or analyzed during the current study are available from the corresponding author on reasonable request.

\section{Ethics approval and consent to participate}

The institutional ethics committee has approved the study (CAAE:

71204717.6.0000.5440). Consent forms were waived due to the retrospective nature of data collection. At enrolment for transplantation, every patient had signed informed consent forms agreeing to participate in HSCT as an experimental protocol, with collection of clinical data and biological samples for the purpose of research.

\section{Consent for publication}

Not applicable.

\section{Competing interests}

The authors declare no financial and non-financial competing interests.

\section{Author details}

${ }^{1}$ Graduate Program in Internal Medicine, Ribeirão Preto Medical School, University of São Paulo, Ribeirão Preto, Brazil. ${ }^{2}$ Center for Cell-Based Therapy, Ribeirão Preto Medical School, University of São Paulo, Ribeirão Preto, Brazil. ${ }^{3}$ Hospital das Clínicas da Faculdade de Medicina de Ribeirão Preto, Ribeirão Preto Medical School, University of São Paulo, Ribeirão Preto, Brazil. ${ }^{4}$ Graduate Program in Oncology, Stem Cells and Cell Therapy, Ribeirão Preto Medical School, University of São Paulo, Ribeirão Preto, Brazil. ${ }^{5}$ Graduate Program in Basic and Applied Immunology, Ribeirão Preto Medical School, University of São Paulo, Ribeirão Preto, Brazil. ${ }^{6}$ Ribeirão Preto School of Nursing, University of São Paulo, Ribeirão Preto, Brazil. ${ }^{7}$ Department of Internal Medicine, Ribeirão Preto Medical School, University of São Paulo, Avenida dos Bandeirantes 3900, Ribeirão Preto, SP 14048-900, Brazil.

Received: 13 November 2020 Accepted: 26 January 2021

Published online: 06 February 2021

References

1. Del Papa N, Pignataro F, Zaccara E, Maglione W, Minniti A. Autologous hematopoietic stem cell transplantation for treatment of systemic sclerosis. Front Immunol. 2018;9:2390. 
2. Henes J, Oliveira MC, Labopin M, Badoglio M, Scherer HU, Del Papa N, et al. Autologous stem cell transplantation for progressive systemic sclerosis: a prospective non-interventional study from the European Society for Blood and Marrow Transplantation Autoimmune Disease Working Party. Haematologica. 2020. https://doi.org/10.3324/haematol.2019.230128.

3. Sullivan KM, Majhail NS, Bredeson C, Carpenter PA, Chatterjee S, Crofford L, et al. Systemic sclerosis as an indication for autologous hematopoietic cell transplantation: position statement from the American Society for Blood and Marrow Transplantation. Biol Blood Marrow Transplant. 2018;24:1961-4.

4. Snowden JA, Badoglio M, Labopin M, Giebel S, McGrath E, Marjanovic Z, et al. Evolution, trends, outcomes, and economics of hematopoietic stem cell transplantation in severe autoimmune diseases. Blood Adv. 2017;1:2742-55.

5. Nash RA, McSweeney PA, Crofford LJ, Abidi M, Chen C-S, Godwin JD, et al. High-dose immunosuppressive therapy and autologous hematopoietic cell transplantation for severe systemic sclerosis: long-term follow-up of the US multicenter pilot study. Blood. 2007;110:1388-96.

6. Binks M, Passweg JR, Furst D, McSweeney P, Sullivan K, Besenthal C, et al. Phase I/I trial of autologous stem cell transplantation in systemic sclerosis: procedure related mortality and impact on skin disease. Ann Rheum Dis. 2001;60:577-84.

7. Burt RK, Oyama Y, Traynor A, Quigley K, Brush M, Rodriguez J, et al. Hematopoietic stem cell transplantation for systemic sclerosis with rapid improvement in skin scores: is neoangiogenesis occurring? Bone Marrow Transplant. 2003;32(Suppl 1):S65-7.

8. Del Papa N, Onida F, Zaccara E, Saporiti G, Maglione W, Tagliaferri E, et al. Autologous hematopoietic stem cell transplantation has better outcomes than conventional therapies in patients with rapidly progressive systemic sclerosis. Bone Marrow Transplant. 2017:52:53-8.

9. Farge D, Marolleau JP, Zohar S, Marjanovic Z, Cabane J, Mounier N, et al. Autologous bone marrow transplantation in the treatment of refractory systemic sclerosis: early results from a French multicentre phase I-II study. $\mathrm{Br}$ J Haematol. 2002;119:726-39.

10. Farge D, Passweg J, van Laar JM, Marjanovic Z, Besenthal C, Finke J, et al. Autologous stem cell transplantation in the treatment of systemic sclerosis: report from the EBMT/EULAR Registry. Ann Rheum Dis. 2004;63:974-81.

11. Henes JC, Schmalzing M, Vogel W, Riemekasten G, Fend F, Kanz L, et al. Optimization of autologous stem cell transplantation for systemic sclerosis -- a single-center longterm experience in 26 patients with severe organ manifestations. J Rheumatol. 2012;39:269-75.

12. Oyama Y, Barr WG, Statkute L, Corbridge T, Gonda EA, Jovanovic B, et al. Autologous non-myeloablative hematopoietic stem cell transplantation in patients with systemic sclerosis. Bone Marrow Transplant. 2007:40:549-55.

13. Burt RK, Oliveira MC, Shah SJ. Cardiac assessment before stem cell transplantation for systemic sclerosis. JAMA. 2014;312:1803.

14. Farge D, Burt RK, Oliveira MC, Mousseaux E, Rovira M, Marjanovic Z, et al. Cardiopulmonary assessment of patients with systemic sclerosis for hematopoietic stem cell transplantation: recommendations from the European Society for Blood and Marrow Transplantation Autoimmune Diseases Working Party and collaborating partners. Bone Marrow Transplant. 2017;52:1495-503.

15. Snowden JA, Sharrack B, Akil M, Kiely DG, Lobo A, Kazmi M, et al. Autologous haematopoietic stem cell transplantation (aHSCT) for severe resistant autoimmune and inflammatory diseases - a guide for the generalist. Clin Med. 2018;18:329-34.

16. Muraro PA, Douek DC, Packer A, Chung K, Guenaga FJ, Cassiani-Ingoni R, et al. Thymic output generates a new and diverse TCR repertoire after autologous stem cell transplantation in multiple sclerosis patients. J Exp Med. 2005;201:805-16.

17. Arruda LC, Clave E, Moins-Teisserenc H, Douay C, Farge D, Toubert A. Resetting the immune response after autologous hematopoietic stem cell transplantation for autoimmune diseases. Curr Res Transl Med. 2016;64:107-13.

18. Arruda LCM, Malmegrim KCR, Lima-Júnior JR, Clave E, Dias JBE, Moraes DA, et al. Immune rebound associates with a favorable clinical response to autologous HSCT in systemic sclerosis patients. Blood Adv. 2018;2:126-41.

19. Assassi S, Wang $X$, Chen G, Goldmuntz E, Keyes-Elstein L, Ying J, et al. Myeloablation followed by autologous stem cell transplantation normalises systemic sclerosis molecular signatures. Ann Rheum Dis. 2019;78:1371-8.

20. Burt RK, Oliveira MC, Shah SJ, Moraes DA, Simoes B, Gheorghiade M, et al. Cardiac involvement and treatment-related mortality after nonmyeloablative haemopoietic stem-cell transplantation with unselected autologous peripheral blood for patients with systemic sclerosis: a retrospective analysis. Lancet. 2013;381:1116-24.

21. Burt RK, Shah SJ, Dill K, Grant T, Gheorghiade M, Schroeder J, et al. Autologous non-myeloablative haemopoietic stem-cell transplantation compared with pulse cyclophosphamide once per month for systemic sclerosis (ASSIST): an open-label, randomised phase 2 trial. Lancet. 2011;378:498-506.

22. van Laar JM, Farge D, Sont JK, Naraghi K, Marjanovic Z, Larghero J, et al. Autologous hematopoietic stem cell transplantation vs intravenous pulse cyclophosphamide in diffuse cutaneous systemic sclerosis: a randomized clinical trial. JAMA. 2014;311:2490-8.

23. Sullivan KM, Goldmuntz EA, Keyes-Elstein L, McSweeney PA, Pinckney A, Welch B, et al. Myeloablative autologous stem-cell transplantation for severe scleroderma. N Engl J Med. 2018;378:35-47.

24. Shouval R, Furie N, Raanani P, Nagler A, Gafter-Gvili A. Autologous hematopoietic stem cell transplantation for systemic sclerosis: a systematic review and meta-analysis. Biol Blood Marrow Transplant. 2018;24:937-44.

25. Kowal-Bielecka O, Fransen J, Avouac J, Becker M, Kulak A, Allanore Y, et al. Update of EULAR recommendations for the treatment of systemic sclerosis. Ann Rheum Dis. 2017:76:1327-39.

26. Oliveira MC, Elias JB, Moraes DA, Simões BP, Rodrigues M, Ribeiro AAF, et al. A review of hematopoietic stem cell transplantation for autoimmune diseases: multiple sclerosis, systemic sclerosis and Crohn's disease. Position paper of the Brazilian Society of Bone Marrow Transplantation. Hematol Transfus Cell Ther. 2020:S2531-1379(20)30032-8.

27. Subcommittee for Scleroderma Criteria of the American Rheumatism Association Diagnostic and Therapeutic Criteria Committee. Preliminary criteria for the classification of systemic sclerosis (scleroderma). Arthritis Rheum. 1980; 23:581-90.

28. van den Hoogen F, Khanna D, Fransen J, Johnson SR, Baron M, Tyndall A, et al. 2013 classification criteria for systemic sclerosis: an American college of rheumatology/European league against rheumatism collaborative initiative. Ann Rheum Dis. 2013;72:1747-55.

29. Clements PJ, Lachenbruch PA, Ng SC, Simmons M, Sterz M, Furst DE. Skin score. A semiquantitative measure of cutaneous involvement that improves prediction of prognosis in systemic sclerosis. Arthritis Rheum. 1990;33:1256-63.

30. Voltarelli JC, Stracieri ABPL, Oliveira MCB, Godoi DF, Moraes DA, Pieroni F, et al. Transplante de células-tronco hematopoéticas em doenças reumáticas. Parte 2: experiência brasileira e perspectivas futuras. Rev Bras Reumatol. 2005:45:301-12

31. Voltarelli JC, Ouyang J. Hematopoietic stem cell transplantation for autoimmune diseases in developing countries: current status and future prospectives. Bone Marrow Transplant. 2003;32(Suppl 1):S69-71.

32. Eyraud A, Scouppe L, Barnetche T, Forcade E, Lazaro E, Duffau P, et al. Efficacy and safety of autologous haematopoietic stem cell transplantation in systemic sclerosis: a systematic review of the literature. Br J Dermatol. 2018;178:650-8.

33. Wada DT, de Almeida FA, de Moraes DA, Dias JBE, Baddini-Martinez J, Oliveira MC, et al. Automatic quantitative computed tomography evaluation of the lungs in patients with systemic sclerosis treated with autologous stem cell transplantation. J Clin Rheumatol. 2020;26(7S Suppl 2):S158-64.

34. Ruaro B, Sulli A, Pizzorni C, Paolino S, Smith V, Alessandri E, et al. Correlations between blood perfusion and dermal thickness in different skin areas of systemic sclerosis patients. Microvasc Res. 2018;115:28-33.

35. Chen $C$, Cheng $Y$, Zhu X, Cai $Y$, Xue $Y$, Kong N, et al. Ultrasound assessment of skin thickness and stiffness: the correlation with histology and clinical score in systemic sclerosis. Arthritis Res Ther. 2020;22:197.

36. Park EH, Strand V, Oh YJ, Song YW, Lee EB. Health-related quality of life in systemic sclerosis compared with other rheumatic diseases: a cross-sectional study. Arthritis Res Ther. 2019;21:61.

37. Costa-Pereira KR, Guimarães AL, Moraes DA, Dias JBE, Garcia J, de Oliveira-Cardoso EA, et al. Hematopoietic stem cell transplantation improves functional outcomes of systemic sclerosis patients. J Clin Rheumatol. 2020;26(75 Suppl 2):S131-8.

38. Farge D, Labopin M, Tyndall A, Fassas A, Mancardi GL, Van Laar J, et al. Autologous hematopoietic stem cell transplantation for autoimmune diseases: an observational study on 12 years' experience from the European Group for Blood and Marrow Transplantation Working Party on Autoimmune Diseases. Haematologica. 2010;95:284-92.

39. Denton CP, Khanna D. Systemic sclerosis. Lancet. 2017;390:1685-99.

40. Burt RK, Han X, Quigley K, Arnautovic I, Shah SJ, Lee DC, et al. Cardiac safe hematopoietic stem cell transplantation for systemic sclerosis with poor cardiac function: a pilot safety study that decreases neutropenic interval to 5 days. Bone Marrow Transplant. 2021;56:50-9.

\section{Publisher's Note}

Springer Nature remains neutral with regard to jurisdictional claims in published maps and institutional affiliations. 